\title{
Die Dialektik des Publizierens in der akademischen Kulturindustrie im Zeitalter der Digitalisierung
}

\author{
Klaus Rummler
}

\section{Von der Publikationsliste zum Leistungsausweis}

In unserem Arbeitsalltag als Wissenschaftlerinnen und Wissenschaftler haben wir es täglich mit Literatur zu tun. Wir empfehlen unseren Studierenden Literatur, geben Leseaufgaben oder kontrollieren Hausarbeiten und achten dabei auf korrekte Zitierweise und die verwendete Literatur. Gleichzeitig schreiben wir selbst Fachartikel, verwenden dabei Literatur, die wir zuvor in unterschiedlichen Nachschlagewerken oder Listen recherchiert haben. Diese Literatur organisieren wir zur besseren Wiederauffindbarkeit, zur leichteren Zitation und zur Annotation in dezidierten Software-Programmen, wie z.B. Citavi, EndNote, Mendeley, oder das Open Source Programm Zotero. Diese Programme helfen dann bei der Erstellung von Literaturlisten für unterschiedliche Zwecke, sei es die Literaturliste für die nächste Lehrveranstaltung als Leseliste, oder für den aktuellen Artikel, den wir gerade schreiben. Eine weitere typische Verwendung ist die Publikationsliste im eigenen Lebenslauf (CV, curriculum vitae).

Gerade wenn es um den eigenen Lebenslauf geht oder, um die Verwaltung von Literatur zu unterschiedlichen Zwecken sind Literaturmanager sehr nützlich. Aber bereits an dem Punkt, wo wir uns als Wissenschaftlerinnen und Wissenschaftler auf eine akademische Position beispielsweise an einer Hochschule bewerben, versuchen Einsitzende einer Auswahlkommission die persönlichen Leistungen anhand der Publikationsliste abzuschätzen. Dort zählen dann neben der Anzahl auch die Frequenz, Titel und Themen der Publikationen sowie Publikationsplattformen und Co-Autor/innenschaften. Für Kolleg/innen aus den Ingenieurs- oder Naturwissenschaften, aber auch für Teile der Humanwissenschaften spielt hier der persönliche Zitationsindex eine tragende Rolle, der für Vertreter/innen der Erziehungswissenschaft, oder der hier vertretenen Perspektive der erziehungswissenschaftlichen Medienpädagogik eine eher untergeordnete Rolle spielt. 
Entsprechend der Diversifizierung der Arbeitsinhalte, den gegebenen Arbeitsstrukturen und der möglichen Datafizierung ist die Publikationsliste allein kein hinreichender Indikator für akademische Leistung. So verlangen z.B. akademische Arbeitgeber, Drittmittelgeber, Fachgesellschaften, Verlage oder Regierungsstellen regelmäßige Berichte von Wissenschaftlerinnen und Wissenschaftlern zu ihren Leistungen. Laut Dagienė und Krapavickaitė (2016: 158) hat rechnerisch und praktisch jede Wissenschaftlerin und jeder Wissenschaftler bereits mindestens einen solchen Bericht abgeliefert - Tendenz steigend. Inhalte dieser Berichte sind Aufstellungen zur bisherigen Bildungs- und Berufskarriere, Wissenschaftspublikationen, Konferenzbeiträgen, Arbeit in Gremien, geleistete Gutachten, Patente, Beratungstätigkeiten (auch gegenüber Studierenden und Promovierenden), Mitgliedschaften in Fachgesellschaften, Forschungs- und Entwicklungsprojekte (einschl. deren eigener Rahmendaten wie Laufzeit, Fördersumme und Fördergeber) plus Aktivitäten in nicht-wissenschaftliche Feldern (vgl. Dagienė und Krapavickaitė 2016: 159).

Angesichts der Qualität dieser Daten, liegt es nahe, diese in digitalen Systemen, sog. Online Research Information Management Systems (vgl. Wu, Stvilia, und Lee 2017), zu verwalten. Viele Hochschulen bieten derartige Systeme mit ihren eigenen Bedürfnissen intern an. Öffentlich zugängliche Beispiele solcher Systeme sind Zotero (Open Source), Mendeley, myEndnoteWeb, Google Scholar, ResearchGate, Academia.edu oder ORCID. Die Minimalfunktion dieser Systeme besteht in der Verwaltung der eigenen Publikationsliste, einschl. einer persönlichen Profilseite, wo diese Daten öffentlich sichtbar sind. Weitere Funktionen können der Upload von Pre-Prints sein, die Auflistung aktueller und abgeschlossener Projekte, oder die Vernetzung mit anderen Wissenschaftlerinnen oder Wissenschaftlern, usw.

Mendeley und EndNote via ResearcherID und ORCID nutzen für den gegenseitigen Transfer dieser Daten eine API-Schnittstelle (application programming interface). Hierdurch ist es prinzipiell möglich, die eigene Publikationsliste direkt im Literaturmanager (z.B. EndNote oder Mendeley) zu bearbeiten und diese mit der Website zu synchronisieren. Das Beispiel ORCID ist in diesem Zusammenhang besonders erwähnenswert, da hier neben der Auflistung der Bildungs- und Berufskarriere, dem Dreiecksverhältnis aus Projekten, Person(en) und Publikationen große Aufmerksamkeit geschenkt wird. Für die einheitliche Darstellung der Bildungs- und Berufskarriere sind in ORCID zunehmend Hochschulen und deren Abteilungen zur Vorauswahl bereitgestellt. Zudem kooperiert ORCID mit zentralen Forschungsförderinstitutionen und bietet Projekte anhand ihrer Fördernummern zur Vorauswahl an. Zusammen mit den bereits vorhandenen ORCID-Profilen und den eingegebenen Publikationen und ihren eindeutigen Identifikatoren (DOI, Digital Object 
Identifier) entsteht daraus ein maschinen-lesbarer Leistungsausweis, der wiederum öffentlich einsehbar, downloadbar und weiter verarbeitbar ist. Der gesamte ORCID-Datensatz für das Jahr 2018 ist verfügbar unter Blackburn u. a. (2018; ca. 38GB als zip, entpackt als XML) sowie die Einführung dazu und Links zu Datensätzen der vergangenen Jahre unter Therese (2018).

Schon jetzt ist seitens einiger Förderinstitutionen wie z.B. dem Schweizerischen Nationalfonds (SNF) zu beobachten, dass bereits bei der Projektbeantragung die $O R C I D$-ID des oder der Antragsteller/in optional abgefragt wird. Es ist zu vermuten, dass mittel- bzw. langfristig die Angabe dieses ORCIDProfils verpflichtend wird und Fördermittelgeber diesen datafizierten Leistungsausweis zur Projektbewertung heranziehen werden. Der digitale Leistungsausweis repräsentiert insofern den bzw. die Wissenschaftler/in nach Außen und ist dadurch mitbestimmend über die $\mathrm{Zu}$ - oder Absage des Projektantrags.

\section{Unsere Publikationen sind Big Data}

...und ermöglichen die einheitliche Formatierung von Zitationen anhand unterschiedlicher Zitationsstile und ermöglichen die Suche nach Literatur in Bibliotheks-katalogen. ${ }^{1}$ Daneben ermöglichen einige von ihnen das Hinzufügen von Literaturmetadaten über eindeutige Identifikatoren wie arXiv, ERIC, Pub$\mathrm{MeD}$, handle, URN ISBN, oder vor allem DOIs (Digital Object Identifier) und deren Verarbeitung im Fließtext des Textverarbeitungsprogramms.

\subsection{Verbreitung akademischer Publikationen mittels Metadaten}

Die meiste Literatur im Wissenschaftsbetrieb wird momentan in Form von Zeitschriftenartikeln produziert. Um diese Artikel auffindbar zu machen und deren Metadaten (Autor/innen, Titel, Erscheinungsjahr, Band, Heft, Seiten und Zeitschrift, etc.) in maschinen-lesbarer Form zu verbreiten, können ihnen diese DOIs vergeben werden, die gleichzeitig als URL aufgelöst werden können und zur Landing-Page des Artikels führen. Analog dazu vergeben einige Verlage wie z.B. transcript oder Springer (einschl. Sub-Verlage) auch DOIs an Artikel in Sammelbänden und Bücher. Dies unterstützt den Mechanismus der Bildung von Zitationsindizes und Verweissystemen, da DOIs regelmäßigen Mustern folgen und daher in digitalen Texten maschinell auffindbar sind.

1 Siehe dazu insbesondere das Open Source Projekt „Citation Style Language (CSL)“ unter https://citationstyles.org/ und https://github.com/citation-style-language. 
Insofern ist es dann auch nützlich, wenn Desktop-Literaturmanager wie EndNote, Mendeley oder Zotero (Open Source), die durch die Nutzer/innen gespeicherte Literatur auf einer online-Plattform spiegeln und öffentlich verfügbar machen. Dies trägt zur Verbreitung von DOIs und ihrer Metadaten bei und erleichtert die Suche nach und den Import von Metadaten in die Literaturmanager z.B. über Webservices wie Google Scholar, Microsoft Research, doaj.org, doar.org, fis-bildung.de, webofscience.com, ScienceDirect.com, JSTOR, usw. (vgl. z.B. Conrad 2017).

Metadaten wissenschaftlicher Literatur werden auch zwischen Webservices auf der Ebene der Server untereinander ausgetauscht. Ein dafür bedeutsamer Mechanismus ist das „Open Archives Initiative Protocol for Metadata Harvesting“ (OAI-PMH) ${ }^{2}$, der es erlaubt, bibliografische Metadaten, die auf Websites und Repositorien im Hintergrund zur Verfügung gestellt werden, zu „ernten“. Diese Erntemaschinen arbeiten ähnlich wie Suchroboter und besuchen relevante Websites in regelmäßigen Zeitabständen, um neue Informationen von den OAI-Schnittstellen zu sammeln und weiter-zuverbreiten. Das OAI-PMH ist ein quelloffener Standard, der es auch finanziell unabhängigen Anbietern, wie z.B. kleinen akademischen Fachzeitschriften ermöglicht, Literatur in bibliografischer Logik zu verbreiten und damit in Bibliothekskatalogen und Discovery Services aufgenommen zu werden. So bietet z.B. das quelloffene Open Journal System (OJS, Public Knowledge Project) ${ }^{3}$ als Betriebsplattform für Fachzeitschriften eine solche OAI-Schnittstelle an.

Aus dem Bereich der kommerziellen Zusammenarbeit zwischen wissenschaftlichen Bibliotheken und Fachverlagen stammen sog. Discovery Services, die vor allem den Datenaustausch zwischen Bibliothekskatalogen sicherstellen. Die vermutlich bedeutendsten Discovery Services werden von den Unternehmen ProQuest ${ }^{4}$ und $E B S C O^{5}$ betrieben. Als eher non-profit bietet das Online Computer Library Center (OCLC) den Discovery Service WorldCat ${ }^{6}$ an, der zugleich eine der wichtigsten Datendrehscheiben für OAI-PMH ist.

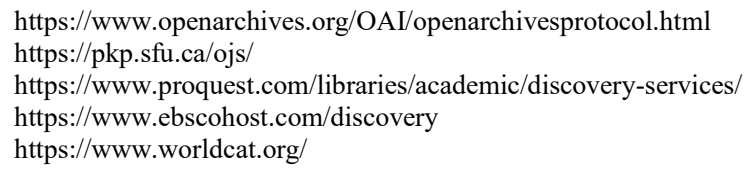




\subsection{Metadaten und Publikationen als wirtschaftlicher Rohstoff für Big Data-Verarbeitung}

Ausgehend von Literaturmanagern ist es nützlich, einen Blick auf wirtschaftliche Verflechtungen und Interessen dieser zu werfen: Abgesehen von der Open Source Software Zotero, deren Finanzierung aus Hochschul- und Stiftungsmitteln ${ }^{7}$ und der Vermarktung zusätzlichen Online-Speichers für angehängte Volltexte stammt, sind die Programme Mendeley und EndNote in der Hand der beiden größten Wissenschaftsverlage. Mendeley startete im Jahr 2008 auf der technischen Basis von Zotero aber mit starkem Fokus auf die Vernetzung mit anderen Wissenschaftler/innen auf der Grundlage gemeinsamer Literatur. Seit dem Jahr 2013 ist Mendeley Teil des Elsevier-Verlags (relX Group) und bietet seine API-Schnittstelle zu den Profilen, zur gespeicherten Literatur bzw. Dokumenten, zu Gruppen und Annotationen öffentlich an. ${ }^{8}$ Elsevier selbst ist einer der größten Anbieter wissenschaftlicher Fachzeitschriften und Buchverlage. Auf der Startseite beschreibt sich das Unternehmen selbst mit den Worten: „Elsevier is a global information analytics business that helps institutions and professionals advance healthcare, open science and improve performance for the benefit of humanity." (https://www.elsevier.com/)

Weitere Produkte im Portfolio umfassen Systeme zur Indexierung und Verbreitung von Fachinformationen. Hierzu gehört u.a. das zuvor unabhängige pre-print Repositorium Social Science Research Network (SSRN) ${ }^{9}$ sowie die Literaturdatenbank Scopus ${ }^{10}$, die zugleich als Indizierungs- und Rankingsystem dient. Aber auch unterschiedliche Systeme zur Messung von Forschungsstrategien und deren Wirkung für unterschiedliche Fachdisziplinen gehören zu Elsevier bzw. zur relX-Gruppe.

Das Programm EndNote ist vermutlich einer der ältesten Literaturmanager, gehörte bis zum Jahr 2016/2017 zum Verlag Thomson-Reuters und ist seit dem Jahr 2017 Teil des Unternehmens Clarivate Analytics. ${ }^{11}$ Laut der Pressemeldung vom 3. Oktober 2016 wurde Clarivate Analytics von Onex Corporation und Baring Private Equity Asia spezifisch dafür gegründet, um jene für Informationsmanagement und -analyse relevanten Produkte von Thomson-Reuters

7 Zitat aus der Fußzeile vom www.zotero.org: ,Zotero is a project of the Corporation for Digital Scholarship and the Roy Rosenzweig Center for History and New Media. It was initially funded by the Andrew W. Mellon Foundation, the Institute of Museum and Library Services, and the Alfred P. Sloan Foundation".

$8 \mathrm{https}: / /$ dev.mendeley.com

9 https://www.ssrn.com/en/index.cfm/ssrn-joins-mendeley-elsevier/

10 https://www.scopus.com

11 Vgl. https://web.archive.org/web/20170110041744/http://clarivate.com/news/ip-and-science-launched-as-independent-company/ 
aufzufangen und eigens zu vermarkten. Bekannte Produkte sind neben EndNote auch die Literaturdatenbank Web of Science bzw. Web of Knowledge. ${ }^{12}$ Das alternative Indizierungssystem Altmetrics gehört seit Anfang 2017 ebenfalls zu Clarivate Analytics ${ }^{13}$ (vgl. Konkiel, Piwowar, und Priem 2014; González-Valiente, Pacheco-Mendoza, und Arencibia-Jorge 2016).

Das Web of Science bzw. die Web of Science Core Collection ${ }^{14}$ - analog zu Scopus von Elsevier - sortiert und hierarchisiert die gelisteten Zeitschriften nach Fachindizes wie z.B. dem Social Sciences Citation Index (SSCI) oder dem Emerging Sources Citation Index (ESCI). Scopus ${ }^{15}$ listet derzeit fast 24.000 aktive und die Web of Science Core Collection über 22.000 Zeitschriften, von denen eine große Anzahl in beiden Indizes vorkommen (vgl. Mering 2017). Die Aufnahme von Zeitschriften in diese Indizes ist über ein strenges Qualitätssicherungssystem geregelt und beinhaltet peer-review Prozesse entlang festgelegter Kriterien. In der Praxis werden die gelisteten Zeitschriften i.d.R. durch kommerzielle Verlage herausgegeben - bestenfalls durch eigene Sub-Verlage dieser beiden großen Anbieter - und erscheinen auf Englisch. Für Zeitschriften und Autor/innen, die ein Interesse an der eigenen Indizierung und an der Teilnahme an akademischem Wettbewerb und Konkurrenzkampf haben, ist die Aufnahme in diese Indizes reizvoll. Die hier gelisteten Zeitschriften gelten als „high ranked“ bzw. überhaupt als ,gerankt“ und versprechen für viele Wissenschaftler/innen hohes Prestige.

Die meisten dieser gerankten Zeitschriften sind entweder Closed Access und werden über Abonnementgebühren finanziert, oder sie verlangen für die Publikation Article Processing Charges (APCs) oder Book Processing Charges (BPCs), um ein Open Access Modell zu finanzieren. Das Directory of Open Access Journals (doaj.org) lässt einen gewissen Rückschluss über die Anzahl an Open Access Zeitschriften zu, die nicht in Scopus oder Web of Science Core Collection gelistet sind. So weist Scopus ca. 4.000 Zeitschriften aus, die in $D O A J$ gelistet sind. DOAJ wiederum weist 3.300 Zeitschriften aus, die APCs verlangen und weist über 9.100 Zeitschriften aus, die keine APCs erheben. ${ }^{16}$ Trotz der Unschärfen und Überlappungen zwischen diesen beiden Verzeichnissen, kann man grob schätzen, dass es ca. 8.000 Zeitschriften gibt, die nicht in Scopus oder Web of Science und in deren Indizes gelistet sind, keine APCs erheben und trotzdem relevant sind, wie z.B. die beiden Zeitschriften MedienPädagogik und Medienimpulse. ${ }^{17}$ Im Verhältnis zur Anzahl der ,gerankten“

12 webofknowledge.com

13 Vgl. https://web.archive.org/web/20170624165834/http://clarivate.com/clarivate-analyticsand-altmetric/

$14 \mathrm{http}: / /$ mjl.clarivate.com/

$15 \mathrm{https}: / / \mathrm{www}$. elsevier.com/?a=91122

16 Vgl. https://doaj.org/search

17 Vgl. die zugehörigen Websites unter www.medienpaed.com und www.medienimpulse.at. 
Zeitschriften macht das immerhin eine Unschärfe von plus/minus 35\% aus. Das heißt, dass bibliometrische oder scientometrische Betrachtungen, die sich lediglich auf Scopus und/oder Web of Science Core Collection beziehen, über ein Drittel aller Zeitschriften ausschließen. Analysen, die nur Scopus und/oder den SSCI einbeziehen, würden sogar noch viel mehr Zeitschriften ausschließen, die aber für den alltäglichen Wissenschaftsdiskurs, vor allem im lokalen Raum bedeutsam sind (weil sie nicht konsequent nur auf Englisch publizieren und durch kleine wissenschaftliche Fachgesellschaften herausgegeben werden und deshalb in keinem dieser Indizes geführt sind). Beispiele solcher Berechnungen und Kritik daran aus Perspektive der Erziehungswissenschaft finden sich z.B. in Buntins et al. (2018) und in Schindler/Rummler (2018).

Die Verschiebungen in der akademischen Publikationslandschaft der vergangenen Jahre und ihre wirtschaftlichen Verflechtungen, wie zuvor exemplarisch dargelegt, zeigen zumindest einen gewissen Trend auf: Die Kernaktivität von Wissenschaftsverlagen liegt nicht nur in der Publikation von Fachartikeln, sondern vielmehr in der Pflege der gesamten Nutzungskette. Dies beginnt mit der Bereitstellung von Literaturverwaltungsprogrammen und dem Sammeln der Daten über verwaltete Literatur und setzt sich mit der Bereitstellung von Literaturdatenbanken mitsamt pre-print Repositorien, einschließlich dem Ranking der in den Datenbanken enthaltenen Literatur fort. Hinzu kommen die Dissemination der Literatur und deren Metadaten in unterschiedliche digitale Systeme bis hin zur statistischen Auswertung all dieser Daten. Allein aus den Pressetexten und Selbstbeschreibungen auf deren Websites kann Leser/in erahnen, dass es den Wissenschaftsverlagen zum einen, um die systematische Auswertung von Metadaten, Nutzungsdaten und in der Konsequenz auch um die maschinelle Auswertung der Volltexte der Publikationen geht. Zum anderen ahnt man bereits bei oberflächlicher Lektüre, dass die Unternehmen diese Auswertungen nicht um des wissenschaftlichen, bibliometrischen oder scientometrischen Selbstzwecks betreiben. Gerade mit der statistischen Bearbeitung der Daten erzielen sie einen beträchtlichen wirtschaftlichen Erlös. Naheliegend und in dieser Überlegung impliziert ist, dass die Unternehmen ihre einzelnen Marken wie Mendeley, Altmetrics, Scopus usw. wie im Fall von Elsevier nicht separat allein operieren lassen, sondern deren Stärken im Hintergrund des Unternehmens verbinden und für einander arbeiten lassen. Das heißt, dass ein beträchtlicher Teil des akademischen Publikationsbetriebs - nachdem wir unsere Beiträge bei den Verlagen eingereicht haben, und Publikationen anderer lesen und zitieren - unter dem Stichwort Big Data zu fassen ist.

Die statistische Auswertung von Metadaten und Nutzungsdaten wissenschaftlicher Literatur ist seit langer Zeit üblich. Erst seit jüngerer Zeit, auch durch deren digitale Verfügbarkeit und deren Erreichbarkeit per Identifikatoren wie DOI (und sie sind Teil der Metadaten), ist die maschinelle Auswertung 
der Volltexte der Publikationen möglich. Einen exemplarischen Hinweis darauf, dass dies bereits betrieben wird, bietet die enge Kooperation zwischen dem Centre for Data Science des University College London (UCL) und Elsevier. Mit der finanziellen Unterstützung von Elsevier wurde dort 2014 die Abteilung UCL Big Data Institute (BDI) ${ }^{18}$ gegründet und zunächst bis 2017 von Patrick Wolfe und dann von Sofia Olhede geleitet. Die Mitglieder und Assoziierten sind erfahrene Wissenschaftler/innen aus unterschiedlichen Fachdisziplinen mit Schwerpunkt Statistik. Die Abteilung ,[...] explores innovative ways to better serve the needs of researchers, through the investigation of new technologies and analytics as applied to scholarly content and data" (https://www.ucl.ac.uk/big-data/bdi). Das BDI listet eine Reihe an Forschungprojekten z.B. mit dem Titel „Investigation of the role and impact of researchers in academic networks" oder "Investigation of relationships between researchers on academic networks". Geleitet werden diese Projekte jeweils von einem der führenden Mitglieder des BDI und zusätzlich von einem Vertreter von Elsevier. Üblicherweise würde man auf der Website einer solchen Abteilung Verlinkungen zu Online-Projektbeschreibungen finden, mit Ausführungen zu Datenmaterial, Erhebungsmethoden, Ablauf und entstandenen Publikationen. Ebenso würde man auf der Ebene der Abteilung üblicherweise Publikationslisten der beteiligten Personen finden. Das Fehlen dieser Informationen ist auffällig und macht stutzig, wo doch gerade Publikationen zum Geschäft und zum Thema dieser Abteilung gehören. Dieses Beispiel führt zur Hypothese, dass die wirklich intensiven und innovativen bibliometrischen Analysen mit Werkzeugen der Big Data-Auswertungen teilweise hinter den verschlossenen Türen der Verlage stattfinden. Die beiden folgenden dokumentierten Beispiele illustrieren einige der aktuell möglichen Big Data-Auswertungen und damit zusammenhängenden Fragestellungen:

Ausgehend von drei verschiedenen wissenschaftstheoretischen, his-torischen Handlungstheorien darüber, wie und warum Autor/innen Zitationen verwenden und insofern aufeinander verweisen (Tahamtan und Bornmann 2018) gehen Bertin et al. (2015) und dann Boyack et al. (2018) einen wesentlichen Schritt weiter in Richtung Big Data-Auswertung wissenschaftlicher Texte. In ihren Untersuchungen betrachten sie die Häufigkeit und Position von Kurzbelegen innerhalb der Textkörper (engl. ,,in-text citations“ im Gegensatz zur Literaturliste am Ende eines Artikels) von über fünf Millionen Volltextartikeln der Datenbanken PubMed Central Open Access Subset und den Elsevier Zeitschriften. Die Autoren vergleichen hinsichtlich der Zitationspraxis fünf Wissenschaftsfelder: „Biomedical and Health Sciences (BHS), Life and Earth Sciences (LES), and Physical Sciences and Engineering (PSE)“ sowie „Mathematics and Computer Science (MCS) and Social Science and Humanities

https://www.ucl.ac.uk/big-data/bdi 
(SSH)“ (ebd.). Für die erste Gruppe stellen sie fest, dass die meisten Kurzbelege im ersten Drittel des Textes, welcher üblicherweise die theoretische Grundlage, Referenzstudien und die Argumentation der Methode enthält, gesetzt werden und schließen daraus, dass in diesen Feldern Methodenartikel häufiger zitiert werden. Für die zweite Gruppe „Mathematics and Computer Science (MCS) and Social Science and Humanities (SSH)“ stellen sie eine gleichmäßigere Verteilung der Kurzbelege über den Text hinweg fest, wobei mehr Kurzbelege bis zur Mitte der Texte zählbar sind und weniger in der zweiten Hälfte. Sie vermuten, dass dies ein Hinweis darauf ist, dass in diesen beiden Feldern stärker auf den Arbeiten anderer Wissenschaftler/innen aufgebaut wird als in den Disziplinen BSE, LHS und PSE. Für alle fünf Felder stellen sie eine Häufung der Anzahl der Zitationen im Zeitraum der vergangenen 15 Jahre fest, wobei die Häufigkeit der Kurzbelege pro Referenz ${ }^{19}$ im Durchschnitt gleich blieb.

Zwischen den Jahren 2015 und 2017 befasste sich eine Forschungsgruppe mit den thematischen Strukturen in 111.616 Artikeln der Astronomie und Astrophysik (Boyack u. a. 2017). Unter dem Titel „Topic Extraction Challenge“ ${ }^{\text {20 }}$ stellt Clarivate Analytics diesen Textkorpus für Wissen-schaftler/innen der Bibliometrie auf Anfrage zur Verfügung. Die konkreten Ergebnisse beider Studien sind wenig eindeutig und für diese Betrachtung eher nebensächlich. Dieses Beispiel verdeutlicht zum einen, dass es seitens z.B. Clarivate Analytics intensive Versuche gibt, Volltextkorpora mit Mitteln der Big Data-Analyse wie z.B. Natural Language Processing, text parsing und mining oder machinelearning zu bearbeiten. Zum anderen verdeutlicht es, dass diese Prozesse im Hintergrund ablaufen, und dass es erst einer Öffnung dieser Datensätze durch die Verlage selbst braucht.

Im Themenheft „Same Data, Different Results“ der Zeitschrift Scientometrics berichtet die Forschungsgruppe über diese „Topic identification challenge“. Neben der Untersuchung der Thematik an sich interessieren im hier diskutierten Zusammenhang vor allem die eingangs formulierten Forschungsfragen:

„Do the sets of publications, authors or institutions we identify and visualise with our methods indeed represent thematic structures? To what extent are results of topic identification exercises determined by properties of knowledge structures, and to what extent are they determined by the approaches we use? Do we produce more than artefacts?" (Gläser et al. 2017)

Ein wesentliches Ergebnis der Studie sind die Unsicherheiten, die in diesem Projekt steckten und die es aufdeckte, sei es aufgrund der Beschaffenheit der 
Rohdaten, die Validität der Operationalisierung der Themen durch die Wahl der Datenmodelle und Algorithmen, die etwaigen Softwarefehler bis hin zu Unsicherheiten in der Interpretation der extrahierten thematischen Strukturen (vgl. Velden u. a. 2017). Sie betonen, dass eine solche Themenanalyse und der Umgang mit damit verbundenen Unsicherheiten von ihrem jeweiligen Zweck abhängen: Soll sie als metrische Analyse einen Beitrag zur Wissenschaftsgeschichte beitragen, soll sie zur Beratung im Diskurs mit Experten beitragen, oder zur Evaluation dienen (vgl. ebd.)?

\section{Akademische Kulturindustrie?}

Die Produktion akademischer Kultur ist fest in wirtschaftlich-industriellen Prozessen und Verflechtungen verankert, verwoben oder vielleicht gar verhaftet. Die digitale Publikationsliste datafiziert den persönlichen Leistungsausweis und ist dann maschinell über API-Schnittstellen als XML oder JSON verarbeitbar. Dieser Prozess der Kulturproduktion kann sich selbst reproduzieren und erhalten. Einer dieser Reproduktionsmechanismen ist die Teilnahme an Sozialen Netzwerken als Kollektiv geteilter Interessen. Mendeley hat dies bereits frühzeitig ausgenutzt und darüber eine breite Masse an Nutzerprofilen akquiriert.

\section{1 „Self-promotion and ego-bolstering “: Akademische Soziale Netzwerke am Beispiel von Academia und ResearchGate}

Zwei weitere solcher sog. „Academic Social Network Sites“ (ASNS) sind Academia.edu und ResearchGate.net. Beide entstanden im Jahr 2008 und erlauben den Upload von Artikeln, pre-Prints, die Angabe von Metadaten zu eigenen Veröffentlichungen als Publikationsliste, die Zuordnung zu Hochschulen und Abteilungen, die Angabe von Projekten sowie das Verbinden mit anderen Nutzenden, dies auch in der Angabe von Ko-Autor/innen der eigenen Veröffentlichungen usw. ResearchGate hat gegenüber Academia mit etwa 15 Millionen registrierten Nutzer/innen ${ }^{21}$ nur etwa ein Fünftel ${ }^{22}$ des Umfangs, hingegen haben sie etwa doppelt so viele Seitenzugriffe ${ }^{23}$. Eine Besonderheit von Academia ist das Anlegen abteilungs- und hochschulspezifischer

23 https://www.similarweb.com/website/researchgate.net versus https://www.similarweb.com/website/academia.edu 
Mikroseiten. Das heißt, dass sich nicht nur Nutzer/innen auf der Ebene der eigenen Hochschule und Abteilung verbinden können, sondern die Hochschule selbst Academia-Seiten verwenden kann, um dort bspw. Publikationslisten zu führen und Leistungsausweise zu bilden. Eine Besonderheit von ResearchGate ist die Entwicklung eines eigenen Reputationsindex (RG), der dann auch spezifisches Interesse in der Bibliometrie und Scientometrie weckt (vgl. Manca 2018: 11; Nicholas et al. 2016).

Das Geschäftsmodell von Academia besteht hauptsächlich aus der Anzeige von Werbung auf den Seiten und in Kampagnen sowie aus bezahlten sog. „Pro“ Accounts von Nutzer/innen. Jenes von ResearchGate baut auf Anzeigen von Werbung, aber laut persönlichen Angaben eines ResearchGate Mitarbeiters gegenüber dem Autor von Anfang 2018 generiert das Unternehmen den Hauptumsatz mit Stellenanzeigen, die Hochschulen dort beauftragen und abonnieren.

In ihrer Literaturanalyse über Artikel, die Academia und ResearchGate thematisieren bzw. analysieren fand Stefania Manca einen Schwerpunkt in den Bibliotheks- und Informationswissenschaften, Informatik und in den Digital Humanities (Manca 2018: 11f.). Hingegen wurden diese ASNS kaum in der Erziehungswissenschaft / Pädagogik oder in der Kommunikationswissenschaft diskutiert, wo doch Soziale Netzwerke in diesen Feldern teilweise intensiv beforscht sind und gerade mit den ASNS das Thema Wissenschaftskommunikation von Interesse sein könnte (ebd.).

Bei der Frage, wozu Wissenschaftler/innen Academia oder ResearchGate nutzen, fanden Meishar-Tal und Pieterse (2017), dass dort hauptsächlich Informationen konsumiert und weniger geteilt werden, und dass noch weniger Interaktion zwischen den Nutzer/innen stattfindet (Meishar-Tal/Pieterse 2017: 17). In Bezug auf die Motivation der Wissenschaftler/innen diese ASNS zu besuchen nennen sie: ,self-promotion and ego-bolstering, acquisition of professional knowledge, belonging to a peer community, and interaction with peers" (ebd.: 17f.). Übersetzt geht es also zunächst um Selbstdarstellung, um $\mathrm{zu}$ wissen, was andere Kolleg/innen beschäftigt und in welchen Zusammenhängen sie aktiv sind und vermutlich auch darum, sich die Anerkennung für die eigene Leistung abzuholen.

Die Kritik an beiden ASNS ist vielfältig. Mehrfach haben Verlage, vor allem Elsevier versucht, die Netzwerke zur Löschung verlagsrechtlich (nicht zu verwechseln mit ,urheberrechtlich“) geschützter Werke zu nötigen. Bei beiden Netzwerken ist auffällig, dass es zwar möglich ist, Metadaten der eigenen Publikationen anzugeben, aber Links und andere Verweise auf den Ablageort der Publikationen sind kaum erkennbar. Die Vermutung liegt daher nahe - und ResearchGate macht das fast aggressiv - dass es diesen Plattformen um den 
Upload der Volltexte geht und eben nicht um die präzise Dokumentation bibliografischer Daten. Es konnten hingegen bislang keine Hinweise gefunden werden, dass Academia oder ResearchGate die dort abgelegten Volltexte nach Big Data auswerten oder zur Auswertung weitergeben. Angesichts der Menge an abgelegten Texten wäre dies aber naheliegend.

Zuletzt zeichnen sich beide Netzwerke durch intensive Kampagnen vor allem per E-Mail aus. Diese E-Mails berichten über Aktivitäten rund um das eigene Profil und um den eigenen Namen, einschl. Meldungen über gefundene Zitationen des eigenen Namens, oder dass wohl eine dritte Person den eigenen Namen in Google suchte usw.

Die Frage, warum sich Wissenschaftler/innen auf ASNS betätigen und vor allem, warum sie dort Artikel und pre-Prints ablegen, bleibt bestehen und sollte weiter beleuchtet werden, da gerade zu diesem Zweck sorgfältigere und zweckgerichtetere Plattformen wie Zenodo.org oder pedocs.de zur Verfügung stehen. Zenodo.org oder pedocs.de haben zwar kaum bzw. keine Netzwerkfunktionen, aber dafür garantieren sie Open Access, Langzeitarchivierung und sind durch öffentliche Stellen und nicht kommerziell finanziert.

Es bleibt also der Eindruck, dass diese Netzwerke primär der Selbstdarstellung und der Generierung von Aufmerksamkeit dienen, eventuell sogar verbunden mit der Hoffnung auf professionellen Erfolg, der sich finanziell auszahlt. Umgekehrt könnte man argumentieren, dass es hierbei auch um gegenseitige soziale Kontrolle geht. Das Beobachten von Stellenanzeigen, das Beobachten neuer Projekte bis hin zu neuen Artikeln innerhalb des eigenen Netzwerks oder der Scientific Community und der eigenen Interessen auf den ASNS gibt Nutzer/innen nützliche Informationen über Kolleg/innen und damit auch über die eigene Positionierung im akademischen Umfeld.

\subsection{Monetarisierung akademischer Leistung}

Im Folgenden scheint es nötig, nochmals aus einer übergeordneten Perspektive wirtschaftliche Interessen und Beziehungen zwischen diesen Institutionen und Unternehmen aufzuzeigen.

Eingangs wurde $O R C I D$ als exemplarische Plattform für die Darstellung von Leistungsausweisen vorgebracht. Bei der Frage nach wirtschaftlichen Verflechtungen wie sie eingangs bereits für die Literaturmanager aufgedeckt wurden, entsteht auch die Frage nach Interessen unterschiedlicher Parteien. 


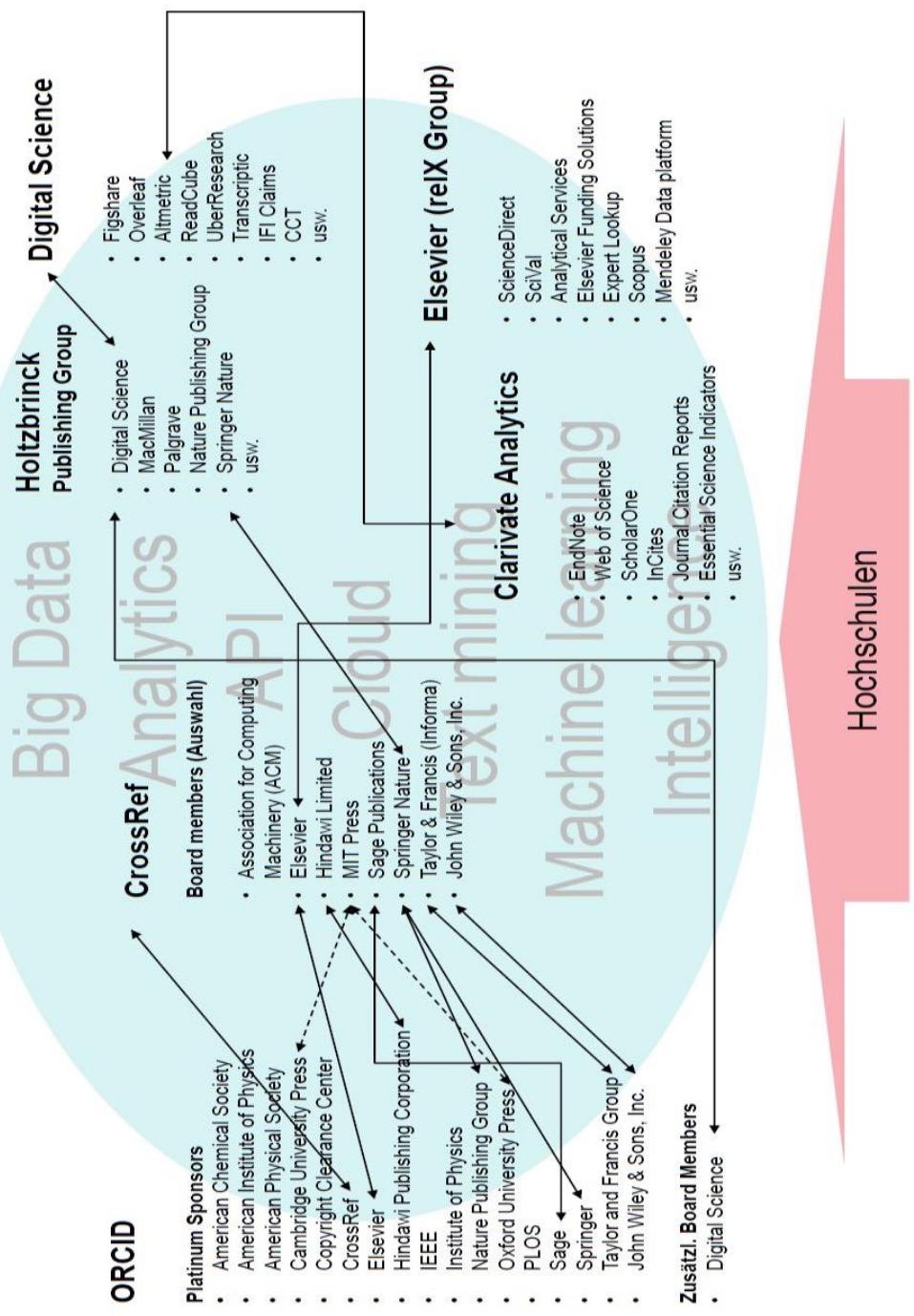

Abbildung 1: Übersicht ausgewählter wirtschaftlicher Verflechtungen (Quelle: Eigene Abbildung)

Im „About ORCID“ “verweisen die Autor/innen darauf, dass ORCID eine ,nonprofit organization" ist, mit dem formulierten Ziel: 
„helping create a world in which all who participate in research, scholarship and innovation are uniquely identified and connected to their contributions and affiliations, across disciplines, borders, and time“ (https://orcid.org/about).

ORCID verweist auf ihrer Website darauf, dass ihr Leitungsgremium, das „Board of Directors“, aus Repräsentant/innen ihrer Mitglieder besteht. Dazu gehörten Anfang 2018 eine Reihe an Platinum Sponsoren, die möglicherweise Aufschluss über wirtschaftliche Verflechtungen geben (vgl. Abb. 1).

Weiter wurden DOIs als eine der tragenden Mechanismen für die digitale Verbreitung bibliografischer Metadaten erklärt. Für Zentraleuropa gibt es drei Institutionen, die DOIs vergeben. Das sind in erster Linie das Unternehmen CrossRef, das Unternehmen MEDRA.de und die non-profit Organisation DataCite. Für die grundlegende Vereinbarung über die Beschaffenheit der Metadaten, die Granularität der Metadaten und die Vereinbarung über einzelne Bezeichner im XML-Schema der DOI-Metadaten wissenschaftlicher Artikel ist CrossRef am bedeutsamsten. MEDRA ist zwar auch auf DOI für wissenschaftliche Fliesstexte spezialisiert, ist aber politisch in der Aushandlung der XML-Schemata nicht maßgeblich. DataCite vergibt DOI hauptsächlich für wissenschaftliche Datensätze, also Forschungsdaten einschl. Software (vgl. Fenner u. a. 2018). Daher lohnt es sich in diesem Kontext die wirtschaftlichen Verflechtungen von Crossref einzubeziehen und auch hier wieder die „Board members" zu betrachten (vgl. Abb. 1).

Abb.1 beschreibt ausgewählte Beziehungen zwischen Hochschulen und wissenschaftsnahen Unternehmen und Verlagen mit ihren metadatenverarbeitenden Diensten und ASNS. Zu den bisher diskutierten großen Verlagen und Unternehmen Elsevier und Clarivate Analytics kommt bei dieser Betrachtung ergänzend die Holtzbrinck Publishing Group aus Stuttgart hinzu, da in ihrem Portfolio weitere bedeutsame Verlage wie Macmillan, Palgrave und Springer Nature enthalten sind.

Zudem ist darin die Unternehmensgruppe Digital Science enthalten, die ihrerseits eine Reihe kleinerer Unternehmen enthält, die besonders auf die Verarbeitung wissenschaftlicher Daten spezialisiert sind wie z.B. FigShare, Overleaf, UberResearch, oder Anteile an Altmetric. Clarivate Analytics, Holtzbrick und Elsevier sind die größten konzernartigen Verlagsgruppen im Wissenschaftsbetrieb. Als unabhängige, in diesem Sinne traditionelle Verlage ohne Überbau in Konzernen kommen die Verlage Taylor \& Francis sowie John Wiley \& Sons dazu. Die beiden letzteren sind Board Members in ORCID und CrossRef (vgl. Abb. 1). Beide der großen unabhängigen Verlage sind insofern direkt an der Formulierung und Dissemination von Metadaten beteiligt und schaffen daher auch die entsprechenden Standards gemeinsam. 
Clarivate Analytics ist über Altmetric als Kooperationsprojekt mit Digital Science an ORCID beteiligt. Elsevier ist sowohl in CrossRef als auch in ORCID direkt beteiligt. Holtzbrick selbst bringt in die Boards von CrossRef und ORCID mindestens Digital Science und Springer Nature ein ${ }^{24}$.

Im Prinzip werden diese Konzerne, Verlage und Institutionen über die Hochschulen finanziert, vor allem wenn man davon ausgeht, dass der große Anteil an Publikationen durch Personen erbracht wird, die an Hochschulen angestellt sind und die Publikationstätigkeit weder als Selbständige betreiben noch zu einem großen Anteil in privatwirtschaftlichen Unternehmen angestellt sind. Bestenfalls können Hochschulen Teile der Personalkosten über Drittmittelgeber wie z.B. die Deutsche Forschungsgemeinschaft (DFG), das Bundesministerium für Bildung und Forschung (BMBF), dem Schweiz-erischen Nationalfonds (SNF), dem Österreichischen Wissenschaftsfonds (FWF), den Programmen der Europäischen Kommission oder dem Europäischen Sozialfonds (ESF) refinanzieren. Seitens der Hochschulen entsteht eine Reihe an Kostenstellen, deren Begünstigte die Verlage und Konzerne sind, z.B.: Abonnementgebühren in den Bibliotheken, Article und Book Processing Charges (APC und BPC), Druckkostenzuschüsse an Verlage und der Rückkauf der ersten Buchauflage. Auch indirekte Kosten entstehen bei der Produktion wissenschaftlicher Publikationen, die vor allem ideeller und weniger materieller Natur sind, aber in Form von Arbeitszeit an die Wissenschaftsarbeiter/innen entrichtet werden. Darunter ist vor allem die Arbeitszeit für das Schreiben von Büchern und Artikeln zu rechnen, aber auch das Layout und das Lektorat, welches wiederum von Verlagen - als bezahlter Service - übernommen werden kann. Die meisten dieser Kosten der Hochschulen werden aus Steuermitteln beglichen, einschl. der Re-finanzierungen über öffentliche Drittmittelgeber, und kommen direkt oder indirekt als Gewinne bei den Verlagen an oder als Gewinne aus Big Data-Auswertungen bei Konzernen wie Clarivate Analytics. Die kritische Frage nach dem finanziellen Return on Investment seitens der Hochschulen, die sich dabei aufdrängt, konnte bislang nicht geklärt werden. In diesem Finanzfluss ist nicht erkennbar, wie und an welcher Stelle Hochschulen ihre Investitionen refinanzieren oder an welcher Stelle Geld aus Publikationstätigkeiten in einer Höhe, die den Investitionen sinnvoll gegenüberstehen, wieder in die Hochschulen zurückfließt. Dieses System ist durchaus vergleichbar mit dem Geschäftsmodell des ersten Zyklus der Abfallwirtschaft (vgl. Bataille und Steinmetz 2014). Auch hier wird der erste kommerzielle Abnehmer oder Verwerter - ähnlich den Verlagen - doppelt bezahlt: Die erste Bezahlung/Vergütung erfolgt, wenn Haushalte ihren Abfall abgeben, entweder direkt über Müllgebühren oder anteilig beim Kauf eines Produktes, und die zweite, wenn 
der Verwerter den Müll - ggf. sortiert und aufbereitet - als Rohstoff weiterverkauft ${ }^{25}$.

Ein weiterer Kostenfaktor seitens der Hochschulen in Richtung der großen Konzerne sind Produkte wie z.B. InCites ${ }^{26}$ von Clarivate Analytics oder Sci$\mathrm{Val}^{27}$ von Elsevier. In diesen Softwareumgebungen zur Berechnung und zum Vergleich institutioneller Produktivität, zum Aufdecken von Kooperationen und Netzwerken sowie zur institutionellen Bewertung akademischer Leistungen kulminiert vermutlich die derzeitige Leistungsfähigkeit der Bibliometrie und Scientometrie anhand der Auswertung großer Datenmengen. In ihnen läuft nicht nur das individuelle Zur-Schau-Stellen akademischer Leistungen von Wissenschaftler/innen zusammen, sondern auch die Ebene der Hochschulen, wo vergleichende Analysen innerhalb und untereinander stattfinden. Bornmann/Haunschild (2017) und Rowlands (2018) üben umfassende Kritik an diesen Entwicklungen. In beiden Beiträgen argumentieren die Autoren, dass die Datenbasis und die Verfügbarkeit bibliometrischer Daten sich in den vergangenen Jahren stark vergrößert hat. Dabei ist auf der einen Seite die Frage, wie diese Daten statistisch am besten auszuwerten wären, einschl., wie mit Unsicherheit umzugehen sei (vgl. Abramo et al. 2016). Lutz Bornmann und Kolleg/innen zeigen mit ihren Arbeiten deutlich auf, dass diese Berechnungen immer noch menschliche Beurteilungen und Einschätzungen benötigen. In ihrem Grundlagenbeitrag haben Bornmann und Daniel (2008) dargelegt, dass Wissenschaftler/innen sich untereinander und ihre Arbeiten nicht nur wegen des fachlichen Gehalts zitieren, sondern dabei auch soziale, nicht-wissenschaftliche Faktoren eine Rolle spielen und eben diese können bibliometrische Verfahren nicht erfassen. Ausgehend vom ursprünglichen Vorhaben der Bibliometrie, die Qualität wissenschaftlicher Arbeit zu erforschen, beobachten die Autoren (2017) einen Wandel hin zu einer rein maschinellen Impact-Messung und fordern, wissenschaftliche Arbeit auch weiterhin - informiert durch Impact-Messung - per Peer-Review zu beurteilen.

„The era of 'desktop bibliometrics' should be welcomed: it promises greater transparency and the opportunity for experimentation in a field that has frankly become a little jaded. The downside is that we are in danger of chasing numbers for numbers' sake, with little understanding of what they mean." (Rowlands 2018)

Die kommerziellen Verlage in den STM-Disziplinen (science, technology, medicine) erzielten im Jahr 2017 ein Marktvolumen von 25,7 Milliarden US-Dollar (Johnson, Watkinson, und Mabe 2018). Dass diese Marktrelevanz nicht allein einer „serial crisis“ der Natur-, Ingenieur- und Humanwissenschaften

25 Siehe dazu auch den Verweis auf den „Müllabfuhr-Streik-Test“ („Garbage Strike Test“) in netzpolitik.org (Dobusch 2016).

26 https://clarivate.com/products/incites/

$27 \mathrm{https}: / /$ www.elsevier.com/solutions/scival 
zuzuschreiben ist haben Larivière/Haustein/Mongeon (2015) beschrieben, indem sie erstmals Marktkonzentrationen der Zeitschriften wenigstens grob nach Fachdisziplinen aufgetrennt haben. Sie zeigten, dass auch in den Sozialwissenschaften einschl. der Erziehungswissenschaft eine beträchtliche Anzahl an vormals unabhängigen Publikationsplattformen wie Zeitschriften oder Hochschulverlagen in großen kommerziellen Verlagen untergekommen sind, mit der Folge, dass auch in dieser Fachgruppe mittlerweile fast ein Drittel aller Artikel über kommerzielle Verlage veröffentlicht wird.

Festzuhalten bleibt, dass es den großen Verlagen gelungen ist, ihr Geschäftsmodell mittels Kundenbindung über z.B. ASNS, Indizes und Big DataAuswertungen nachhaltig auszuweiten und zu stabilisieren. Die Beispiele oben zeigen, dass Verlage auch oder gerade mit Open Access, das ursprünglich als disruptiv angesehen wurde, ebenfalls erfolgreich in ihr Geschäftsmodell integrieren konnten. Eine weiterhin offene Frage bleibt, welche Rolle das Individuum der/des Wissenschaftlerin/s in diesem System spielt und wie wir die beschriebenen Entwicklungen aus Sicht der Erziehungswissenschaft oder der Medienpädagogik - auch aus institutioneller Sicht - bewerten.

\section{Medienpädagogisches Fazit: Akademisches Publizieren als industrielle Kulturproduktion - ein Massenbetrug?}

Aus Sicht der Medienpädagogik stecken wir beim Thema akademisches Publizieren in einem beträchtlichen Dilemma. So argumentiert Dander (2018) zunächst für ein medienpädagogisches „Konzept von Datenkritik, das Reflexion und Handeln, data literacy und Online Privacy Literacy zusammenführt" und eröffnet damit zugleich das auch hier vorliegende Dilemma, nämlich die Vernachlässigung größerer Zusammenhänge und eines allgemeinen Medialitätsbewusstseins sowie einer ,grundlegenden Absage an die Vorstellung des souveränen Subjekts“" (Dander 2018: 99). Konkret bedeutet das hier, dass wir uns als Wissenschaftler/innen darüber bewusst sein müssen, dass mit Publikationen gleichzeitig unsere personenbezogenen Daten weitergegeben werden ob wir wollen oder nicht. Da würden wir als Medienpädagog/innen einerseits warnen, so wenig personenbezogene Daten wie möglich von uns zu geben, und würden auf der anderen Seite akademische Leistungsdaten, angefangen bei der Publikationsliste und der institutionellen Zugehörigkeit einschl. der EMailadresse auf diversen Plattformen weitergeben. Und selbst wenn wir versuchen würden dies zu vermeiden, wäre die Befürchtung keine akademische Anerkennung mehr erringen zu können. Selbst ein vorsichtiger Mittelweg, also 
lediglich in Platinum Open Access Zeitschriften unter einer Creative Commons Lizenz zu publizieren, würde nicht davor schützen, dass personenbezogene Daten weitergeben werden und die Volltexte der eigenen Arbeiten in Big Data-Mechanismen verarbeitet werden würden.

Weitere Dilemmata bringen die Anstellungsverhältnisse an Hochschulen mit sich. Hier werden teilweise Anzahlen von Publikationen in individuellen Leistungsvereinbarungen festgeschrieben. Bestimmte Wissenschaftsdisziplinen bedeuten gleichzeitig in bestimmten ,high-ranked“ Zeitschriften zu veröffentlichen oder in bestimmten Buchverlagen $\mathrm{zu}$ veröffentlichen (vgl. Schindler/Rummler 2018). Auch mit den Mitteln der Biblio- und Scientometrie wäre das Verhältnis zwischen individualisierter Leistungsdarstellung und domänenspezifischer Themen- und Diskusentwicklung weiterhin zu untersuchen. Dabei ginge es um das Verhältnis zwischen dem Publizieren für die eigene Publikationsliste und der Frage danach, ob nicht vielmehr Fachdiskurse und ihre Entwicklung in kleinen, regionalen Ebenen stattfinden (vgl. Rummler 2016, 2017). Dahinter stecken zwei Vermutungen. Zum einen, dass Publikationen in ,high-ranked“ Zeitschriften, zumindest in der Erziehungswissenschaft oder in der Medienpädagogik, nicht notwendigerweise einen Beitrag zur jeweils aktuellen Fachentwicklung beitragen. Dies gilt wahrscheinlich auch z.B. für einen je spezifisch britisch, skandinavisch, oder deutsch-sprachigen Raum. Zum anderen steckt darin die Vermutung, dass auch das unabhängige Publikationswesen die eigenen Potenziale zunächst besser ausnutzen und gleichzeitig neu ausrichten muss. Das beinhaltet z.B. (a) eine konsequentere Nutzung der Platinum Open Access Publikationsplattformen, dabei gleichzeitig (b) die konsequente Nutzung der Verbreitung bibliografischer Metadaten, um gleichzeitig (c) einen engen und zeitnahen Fachdiskurs zu führen.

Das akademische Publizieren ist industrielle Kulturproduktion; sie ist vergleichbar mit einer Kulturindustrie (vgl. Horkheimer und Adorno 1944). Die Akteure darin werden reduziert, bzw. reduzieren sich selbst auf datafizierte Leistungsausweise, die massenhaft innerhalb dieser Kulturindustrie gehandelt werden. Mit der Masse der produzierten Kultur, nämlich der wissenschaftlichen Beiträge, zusammen mit den Anstellungsverhältnissen sind dieser Kultur auch noch die Rezipienten abhandengekommen. Die Rezipienten sind in diesem Kreislauf gleichzeitig die Autor/innen und somit die Produzent/innen. Sie sind die Arbeiter/innen - und ihre Produktionsstätten/Fabrikshallen sind die Hochschulen. Doch an dieser Stelle löst sich die Trennung von Arbeit und Kapital auf, denn das Kapital liegt nicht mehr bei den Arbeitgebern, die wiederum zwar die Kulturindustrie reproduzieren, dies aber, indem sie Steuergelder zum neuen Kapital verschieben, nämlich zu den großen Verlagen und Konzernen.

Ausblickend wäre es durchaus sinnvoll, auch die Erziehungswissenschaft und ihre Sub-Disziplinen mit bibliometrischen Mitteln zu untersuchen, und 
zwar nicht nur durch externe Fachpersonen wie es bereits z.B. Dees/Botte (2013) oder Bambey (2016) zeigten, sondern durch die eigenen Kolleg/innen innerhalb der Scientific Community wie es Buntins et al. (2018) ansatzweise zeigten. Dann gilt es fachgruppenspezifische Fragestellungen zu entwickeln, die es erlauben, z.B. vorsichtig auch nach dem Impact der Medienpädagogik zu fragen; danach zu fragen, wie wir die eigene Forderung nach Open Access und Open Educational Practices (DGfE Sektion Medienpädagogik 2017; Deimann 2019) einlösen und wie es uns insgesamt gelingt, dies sinnvoll in einen Rahmen von Wissenschaftskommunikation einzuflechten.

\section{Literatur}

Abramo, Giovanni/ Ciriaco D'Angelo, Andrea /Grilli, Leonardo (2016): From Rankings to Funnel Plots: The Question of Accounting for Uncertainty When Assessing University Research Performance. In: Journal of Informetrics 10, 3, S. 854-62. DOI: https://doi.org/10.1016/j.joi.2016.07.005.

Bambey, Doris (2016): Fachliche Publikationskulturen und Open Access. Fächerübergreifende Entwicklungstendenzen und Spezifika der Erziehungswissenschaft und Bildungsforschung. Dissertation, Darmstadt: Technische Universität Darmstadt. http://nbn-resolving.de/urn:nbn:de:tuda-tuprints-56032 [Zugriff: 5.11.2019].

Bataille, Mar/Steinmetz, Alexander (2014): Kommunale Monopole in der Hausmüllentsorgung. In: DICE Ordnungspolitische Perspektiven 63. Düsseldorf: Düsseldorf Institute for Competition Economics (DICE). http:/hdl.handle.net/10419/ 100635 [Zugriff: 5.11.2019].

Bertin, Marc/Atanassova, Iana/Gingras, Yves/Larivière, Vincent (2015): The Invariant Distribution of References in Scientific Articles: The Invariant Distribution of References in Scientific Articles. In: Journal of the Association for Information Science and Technology 67, 1, S. 164-77. DOI: https://doi.org/10.1002/asi.23367.

Blackburn, Rob/ Brown, Josh/ Buys, Matthew/Calvo, Manuel/Cardoso, Ana/Demain, Paula/Demeranville, Tom, u. a. (2018): ORCID Public Data File 2018. DOI: https://doi.org/10.23640/07243.7234028.v1.

Bornmann, Lutz/Daniel, HansDieter (2008): What Do Citation Counts Measure? A Review of Studies on Citing Behavior. In: Journal of Documentation 64, 1 S. 45-80. DOI: https://doi.org/10.1108/00220410810844150.

Bornmann, Lutz/Haunschild, Robin (2017): Quality and Impact Considerations in Bibliometrics: A Reply to Ricker (in Press). In: Scientometrics 111, 3, S. 1857-59. DOI: https://doi.org/10.1007/s11192-017-2373-3.

Boyack, Kevin/ Glänzel, Wolfgang/Gläser, Jochen/ Havemann, Frank/ Scharnhorst, Andrea/Thijs, Bart/van Eck, Nees Jan/Velden, Theresa/Waltmann, Ludo (2017): Topic Identification Challenge. In: Scientometrics 111, 2, S. 1223-24. DOI: https://doi.org/10.1007/s11192-017-2307-0. 
Boyack, Kevin W./van Eck, Nees Jan/Colavizza, Giovanni/Waltman, Ludo (2018): Characterizing In-Text Citations in Scientific Articles: A Large-Scale Analysis. In: Journal of Informetrics 12, 1, S. 59-73. DOI: https://doi.org/10.1016/j.joi.2017. 11.005 .

Buntins, Katja/Bedenlier, Svenja/Bond, Melissa/Kerres, Michael/Zawacki-Richter, Olaf (2018): Mediendidaktische Forschung aus Deutschland im Kontext der internationalen Diskussion. Eine Auswertung englischsprachiger Publikationsorgane von 2008 bis 2017. In: Getto, Barbara/ Hintze, Patrick/Kerres, Michael (Hrsg.): Digitalisierung und Hochschulentwicklung: Proceedings zur 26. Tagung der Gesellschaft für Medien in der Wissenschaft e.V. Münster New York: Waxmann, S. 246-63. https://www.waxmann.com/?eID=texte\&pdf=3868Volltext.pdf\&typ=zusatztext [Zugriff: 5.11.2019].

Conrad, Lettie Y. (2017): Headlines from the Discovery Files: Key Publications on Scholarly Content Discoverability: Scholarly Discovery Headlines. In: Learned Publishing 30, 1, S. 31-37. DOI: https://doi.org/10.1002/leap.1080.

Dagienė, Eleonora/Krapavickaitè, Danuté (2016): How Researchers Manage Their Academic Activities. In: Learned Publishing 29, 3, S. 155-63. DOI: https://doi.org/ 10.1002/leap.1030.

Dander, Valentin (2018): Medienpädagogik im Lichte | im Schatten digitaler Daten. Manteltext. In: Dander, Valentin (Hrsg.): Zurück in die Zukunft der Medienpädagogik. «Subjekt», «Bildung» und «Medien*Kritik» im Lichte | im Schatten digitaler Daten, S. 1-134. Zürich: MedienPädagogik: Zeitschrift für Theorie und Praxis der Medienbildung. DOI: https://doi.org/10.21240/mpaed/diss.vd.01.X.

Dees, Werner/Botte, Alexander (2013): Publikationspraxis in der Bildungsforschung 1998-2007. Frankfurt a. M.: pedocs. http://nbn-resolving.de/urn:nbn:de:0111opus-70473 [Zugriff: 5.11.2019].

Deimann, Markus (Hrsg.) (2019): Forschung und Open Educational Resources - Eine Momentaufnahme für Europa. Bd. 34. Themenhefte. Zürich: MedienPädagogik: Zeitschrift für Theorie und Praxis der Medienbildung. DOI: https://doi.org/ $10.21240 / \mathrm{mpaed} / 34$.X.

DGfE Sektion Medienpädagogik (2017): Orientierungsrahmen für die Entwicklung von Curricula für medienpädagogische Studiengänge und Studienanteile. In: MedienPädagogik: Zeitschrift für Theorie und Praxis der Medienbildung, Dezember, 17. DOI: https://doi.org/10.21240/mpaed/00/2017.12.04.X.

Dobusch, Leonhard (2016): Deutsche Universitäten 2017 im digitalen Ausnahmezustand: Kämpfen oder Kapitulieren? netzpolitik.org (blog). 6. Dezember 2016. https://netzpolitik.org/2016/deutsche-universitaeten-2017-im-digitalen-ausnahmezustand-kaempfen-oder-kapitulieren/ [Zugriff: 5.11.2019].

Fenner, Martin/Katz, Daniel S./Holm Nielsen, Lars/Smith, Arfon (2018): DOI Registrations for Software, Mai. DOI: https://doi.org/10.5438/1nmy-9902.

Gläser, Jochen/Glänzel, Wolfgang/Scharnhorst, Andrea (2017): Same Data -Different Results? Towards a Comparative Approach to the Identification of Thematic Structures in Science. In: Scientometrics 111, 2, S. 981-98. DOI: https://doi.org/ 10.1007/s11192-017-2296-z. 
González-Valiente/Luis, Carlos/Pacheco-Mendoza, Josmel/Arencibia-Jorge, Ricardo (2016): A Review of Altmetrics as an Emerging Discipline for Research Evaluation: A Review of Altmetrics. In: Learned Publishing 29, 4, S. 229-38. DOI: https://doi.org/10.1002/leap.1043.

Horkheimer, Max/Adorno, Theodor W. (1944): Dialektik der Aufklärung: philosophische Fragmente. Frankfurt a. M.: Fischer-Taschenbuch-Verl.

Johnson, Rob/Watkinson, Anthony/Mabe, Michael (2018): The STM Report. An overview of scientific and scholarly publishing. International Association of Scientific, Technical and Medical Publishers. https://www.stm-assoc.org/2018_10_04_ STM_Report_2018.pdf [Zugriff: 5.11.2019].

Konkiel, Stacy/Piwowar, Heather/Priem, Jason (2014): The Imperative for Open Altmetrics. In: The Journal of Electronic Publishing 17, 3 https://doi.org/10.3998/ 3336451.0017.301.

Larivière, Vincent/Haustein, Stefanie/Mongeon, Philippe (2015): The Oligopoly of Academic Publishers in the Digital Era. Wolfgang Glanzel (Hrsg.): PLOS ONE 10, 6: e0127502. DOI: https://doi.org/10.1371/journal.pone.0127502.

Manca, Stefania (2018): ResearchGate and Academia.edu as networked socio-technical systems for scholarly communication: a literature review. Research in Learning Technology 26 (Februar). DOI: https://doi.org/10.25304/rlt.v26.2008.

Meishar-Tal/Hagit/Pieterse, Efrat (2017): Why Do Academics Use Academic Social Networking Sites? In: The International Review of Research in Open and Distributed Learning 18, 1. DOI: https://doi.org/10.19173/irrodl.v18i1.2643.

Mering, Margaret (2017): Bibliometrics: Understanding Author-, Article- and JournalLevel Metrics. In: Serials Review 43, 1, S. 41-45. DOI: https://doi.org/10.1080/ 00987913.2017.1282288.

Nicholas, David/Clark, David/Herman, Eti (2016): ResearchGate: Reputation Uncovered: ResearchGate: Reputation Uncovered. In: Learned Publishing 29, 3, S. $173-$ 82. DOI: https://doi.org/10.1002/leap.1035.

Rowlands, Ian (2018): What Are We Measuring? Refocusing on Some Fundamentals in the Age of Desktop Bibliometrics. In: FEMS Microbiology Letters 365, 8. DOI: https://doi.org/10.1093/femsle/fny059.

Rummler, Klaus (2016): Conferences In The Context Of Academic Performance, Informal Learning And Alternative Designs - Between Theory And Practice. Roundtable gehalten auf der ECER 2016 Leading Education: The Distinct Contributions of Educational Research and Researchers, University College Dublin, August 24. http://www.eera-ecer.de/ecer-programmes/conference/21/contribution/38886/ [Zugriff: 5.11.2019].

Rummler, Klaus (2017): Structures of Academic Knowledge Distribution and Academic Identity Management. Panel Discussion gehalten auf der ECER 2017: Reforming Education and the Imperative of Constant Change: Ambivalent roles of policy and the role of educational research, University College Copenhagen, August 22 .

Schindler, Christoph/Rummler, Klaus (2018): Open Access in der Publikationslandschaft der Erziehungswissenschaft. Eine Sondierung mit Blick auf Monographien und Sammelwerke. Erziehungswissenschaft 29, 57, S. 8-18. DOI: https://doi.org/10.3224/ezw.v29i2.02. 
Tahamtan, Iman/Bornmann, Lutz (2018): Core Elements in the Process of Citing Publications: Conceptual Overview of the Literature. In: Journal of Informetrics 12, 1, S. 203-16. DOI: https://doi.org/10.1016/j.joi.2018.01.002.

Therese, Alainna (2018): Celebrating Open Access Week with the 2018 Public Data File. In: Text. 24. Oktober 2018. https://orcid.org/blog/2018/10/24/2018-publicdata-file [Zugriff: 5.11.2019].

Velden, Theresa/Boyack, Kevin W./Gläser, Jochen/Koopman, Rob/Scharnhorst, Andrea/Wang, Shenghui (2017): Comparison of Topic Extraction Approaches and Their Results. In: Scientometrics 111, 2, S. 1169-1221. DOI: https://doi.org/10. 1007/s11192-017-2306-1.

Wu, Shuheng/Stvilia, Besiki/Joon Lee, Dong (2017): Readers, Personal Record Managers, and Community Members: An Exploratory Study of Researchers' Participation in Online Research Information Management Systems. In: Journal of Library Metadata 17, 2, S. 57-90. DOI: https://doi.org/10.1080/19386389. 2017.1348783. 